\title{
Taurine Positively Affects Rabbit Spermatozoa Quality in Vitro
}

Peter Massanyi ${ }^{1 *}$, Filip Tirpak ${ }^{1}$, Tomas Slanina ${ }^{1}$, Peter Massanyi Jr. ${ }^{2}$, Marko Halo Jr. ${ }^{1}$, Martin Massanyi ${ }^{1}$, Jan Danko ${ }^{2}$, Robert Stawarz ${ }^{3}$ and Norbert Lukac ${ }^{1}$

1. Slovak University of Agriculture in Nitra, Nitra, Slovak Republic

2. University of Veterinary Medicine and Pharmacy in Kosice, Kosice, Slovak Republic

${ }^{3}$ Pedagogical University of Krakow, Krakow, Poland

* Corresponding author: massanyip@gmail.com

Taurine has been identified as a non-permeating spermatozoa cryoprotectant which minimizes the cellular damage [1]. Intracellular taurine is present in cells at high amounts and its role is determined by the cell type. The use of taurine in reproduction comes out of ability to sustain the spermatozoa motility and to take part in spermatozoa capacitation [2]. The addition of anti-oxidants, especially those which are contained in epididymal and oviductal fluids (e.g., taurine), to semen extenders protects the spermatozoa against the ROS generated during freezing and thawing process $[1,3]$. The aim of this study was to determinate the effect of various taurine concentrations on rabbit spermatozoa in in vitro conditions. Taurine, amino acid distinguished by its strong antioxidant properties, has been widely tested on spermatozoa of all kinds of animals except for the rabbits. This study investigated the effects of taurine on motility and viability on rabbit spermatozoa. The control sample and samples enriched with taurine solutions - $3.125 \mathrm{mM}, 6.250 \mathrm{mM}$ and $12.5 \mathrm{mM}$ were analyzed using microscopic methods [4]. Ejaculates of nine adult rabbits were subjected to this study and observed at time intervals 0, 90, 180 and 270 minutes while cultured at $39^{\circ} \mathrm{C}$. For detection of viability the mitochondrial toxicity test (MTT) was used and motility parameters were carried out using computer assisted semen analysis (CASA). Experimental samples were compared against the control. Monitored motility parameters (motility, progressive motility and velocity curved line of spermatozoa) showed that taurine treated spermatozoa have lower activity at the beginning of the study but along with increasing time the positive effect of taurine was induced.

Significant $(\mathrm{P}<0.05)$ values were observed in velocity curved line in time intervals $0(3.125 \mathrm{mM}-$ negative effect: $110.6 \pm 26.85 \mu \mathrm{m} / \mathrm{s})$ and $180(12.5 \mathrm{mM}$ - positive effect: $105.8 \pm 28.36 \mu \mathrm{m} / \mathrm{s})$. Assessment of the mitochondrial toxicity revealed that the addition of taurine showed the higher viability in supplemented samples however with no statistical significance. Results indicate that taurine antioxidant properties maintain the spermatozoa viability and activity. Generally, it can be concluded, that taurine supplemented samples had lower activity in inceptive time intervals.

\section{References:}

[1] MN Bucak and N Tekin, N., Small Ruminant Research 73 (2007), p. 103.

[2] S Chhillar, VK Singh, R Kumar and SK Atreja, Animal Reproduction Science 135 (2012) p. 1.

[3] S Sariozkan et al., Cryobiology 58 (2009), p. 134.

[4] F Tirpak et al., Slovak Journal of Animal Science 48 (2015), p. 49.

[5] This work was supported by projects APVV-16-0289, VEGA 1/0539/18, and KEGA 010/SPU-4/2018. 